\title{
Measurement Error Analysis of Volumetric Gas Meter Without Temperature Compensation
}

\author{
Yin Xiao, Tian Guansan", Zhang Wenhao \\ School of Thermal Engineering, Shandong Jianzhu University, Jinan, China \\ Email address: \\ Y686997x@163.com (Yin Xiao), tgs4170@sdjzu.edu.cn (Tian Guansan), 17865312572@163.com (Zhang Wenhao) \\ ${ }^{*}$ Corresponding author
}

To cite this article:

Yin Xiao, Tian Guansan, Zhang Wenhao. Measurement Error Analysis of Volumetric Gas Meter Without Temperature Compensation. International Journal of Economy, Energy and Environment. Vol. 6, No. 5, 2021, pp. 118-123. doi: 10.11648/j.ijeee.20210605.17

Received: September 10, 2021; Accepted: October 8, 2021; Published: October 19, 2021

\begin{abstract}
There is a certain production and sales difference in the urban gas transmission and distribution system, which is composed of many factors. Part of the reason is that the gas meter takes the volume under standard conditions as the measurement unit, but the gas temperature and pressure in the meter change with the environment of the gas supply pipelines, resulting in the measurement error of the domestic gas meter without temperature and pressure compensation. By analyzing the change of annual gas temperature and local atmospheric pressure when outdoor courtyard pipelines are overhead and buried in a city, the influence of temperature and pressure change on gas volume is discussed by using gas state equation, the production and sales difference caused by metering error is calculated, and the corresponding correction coefficient is put forward, which is of certain significance to reduce the production and sales difference. It is concluded that when temperature of the gas is lower than $20^{\circ} \mathrm{C}$, the measurement error of the gas meter is negative, which has caused the loss of the gas company. For domestic gas meters without temperature compensation, the product of measured gas consumption and temperature correction coefficient can be taken as the actual gas consumption to correct the measurement error of gas meters.
\end{abstract}

Keywords: Volumetric Gas, Temperature, Pressure, Correction Factor, Measurement Error

\section{Introduction}

In the process of gas sales, the standard condition volume is taken as the measurement unit. During measurement, the standard reference conditions of temperature and pressure are $101325 \mathrm{~Pa}, 20^{\circ} \mathrm{C}(293.15 \mathrm{~K})$, the gas volume under this temperature and pressure is taken as the standard volume [1]. However, due to the spacing between gas molecules and the compressibility and thermal expansion characteristics of gas, the gas volume is affected by temperature and pressure. When there is a difference between the actual state and the measurement standard state and the gas meter does not have temperature and pressure compensation, the measurement error will be caused. Resident users consume less gas, so most gas metering uses diaphragm gas meters without temperature and pressure compensation. Many gas companies have paid attention to and studied the measurement error of gas meters for residential users. Hangzhou Gas Company installed a temperature collector at the screw plug of the inlet pipe in front of the building, measured the gas temperature every month, and calculated that the measurement loss of the gas meter due to no temperature and pressure compensation in one year was $152.36 \times 10^{4} \mathrm{~m}^{3}$, accounting for $1.29 \%$ of the annual gas supply and marketing difference [2]. Beijing gas group collects and analyzes the annual natural gas temperature, calculates that the annual measurement error caused by no temperature and pressure compensation is - $3.4 \%$ [3]. Reference [4] introduces two methods for evaluating the uncertainty of indication error of diaphragm gas meter. Through comparison, the role of sensitivity coefficient in the evaluation of relative uncertainty is introduced. In reference [5], the metering performance of temperature compensated membrane gas meter is tested. Reference [6] proposes a relative error calibration technology according to the near-infrared communication protocol through the optical interface. By improving the structure, canceling the mechanical counter and adding the optical interface, the preliminary calibration, correction and recalibration are 
implemented through pulse equivalent to realize the automatic calibration. This paper considering the influence of local atmospheric pressure, gas supply pressure and gas temperature in the pipeline under different laying conditions on measurement error, taking J City as an example, analyzes and calculates the gas loss caused by measurement error.

\section{Metering Principle of Gas Meter}

At present, membrane gas meters are mostly used in domestic and catering gas measurement. The gas volume measurement is carried out by driving the left and right movement of the airbag endothelial membrane through the pressure difference between the inlet and outlet of the gas meter. The structure of gas meter is shown in Figure 1.

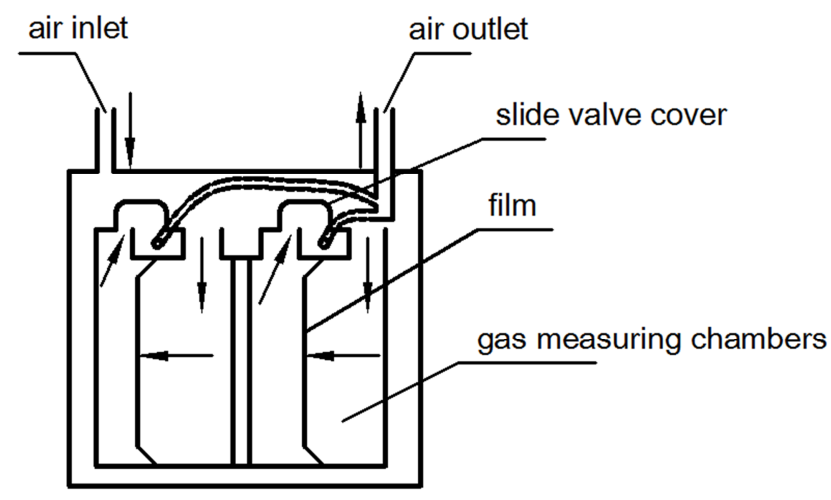

Figure 1. Structure of gas meter.

There are two gas measuring chambers in the gas meter. Each measuring chamber is divided into left and right chambers by the film. The film moves left and right. The connecting rod mechanism is driven by the rocker to control the rotary movement of the slide valve cover. The slide valve cover can cover the air inlet of the left and right chambers respectively. The chamber covered by the slide valve cover can only be connected with the air outlet. The air inlet and outlet of the gas measuring chamber can be changed by the movement of the slide valve cover. When in use, the user opens the valve in front of the stove, the original gas in the small chamber covered by the slide valve cover flows out from the air outlet, and the pressure decreases. The gas flows into the chamber not covered by the slide valve cover from the air inlet to push the film to move to the chamber with reduced pressure. When the film moves to a certain position, that is, when the air inlet chamber is filled with gas, the rocker end connected with the film and the swing rod fixed with the vertical shaft of the rocker swing, which drives the connecting rod crank and the sliding valve at the inlet and outlet of the metering chamber to move. The slide valve cover rotates and the covered chamber opens, Start air intake; On the contrary, the chamber filled with gas on the other side is covered and compressed by the skin film, and the gas is discharged from the air outlet. Each time the chamber in the gas meter completes the air inlet and outlet, that is, when the membrane moves from the left limit position to the right limit position, it completes the measurement. The counter records as "one rotation volume", which is the main object of gas meter measurement and the most basic unit of membrane gas meter measurement [7].

\section{Annual Gas Temperature}

\subsection{Gas Inlet Temperature of Gas Meter}

The gas inlet temperature is the main factor that affects the measurement error of the gas meter. Gas is transported by pipelines and enters users through gas meters. Gas pipelines can be divided into outdoor courtyard pipelines and indoor user pipelines. The user pipelines are directly connected to the gas meters, but the length of the pipeline is short and the gas flow rate is fast. The heat transfer is small, so the gas temperature in the courtyard pipelines is regarded as the inlet temperature. The length of outdoor courtyard gas pipelines is long and no insulation layer. The gas conducts heat transfer with the surrounding environment. The gas temperature in the pipelines is basically equal to the environment temperature where the pipelines located. There are two kinds of laying methods for courtyard pipelines, namely, buried and overhead. The gas temperature in the overhead pipelines can be regarded as equal to the air temperature. The gas in the buried pipelines achieved thermal equilibrium through heat transfer with the surrounding soil. The temperature is approximately equal to the soil temperature [8].

\subsection{Gas Temperature in Overhead Courtyard Pipeline}

As a typical climate city in the north, city $\mathrm{J}$ is cold in winter and hot in summer, with large annual temperature difference. The meteorological temperature of $8760 \mathrm{~h}$ in $\mathrm{J}$ city is obtained from the meteorological department, and the change is shown in Figure 2.

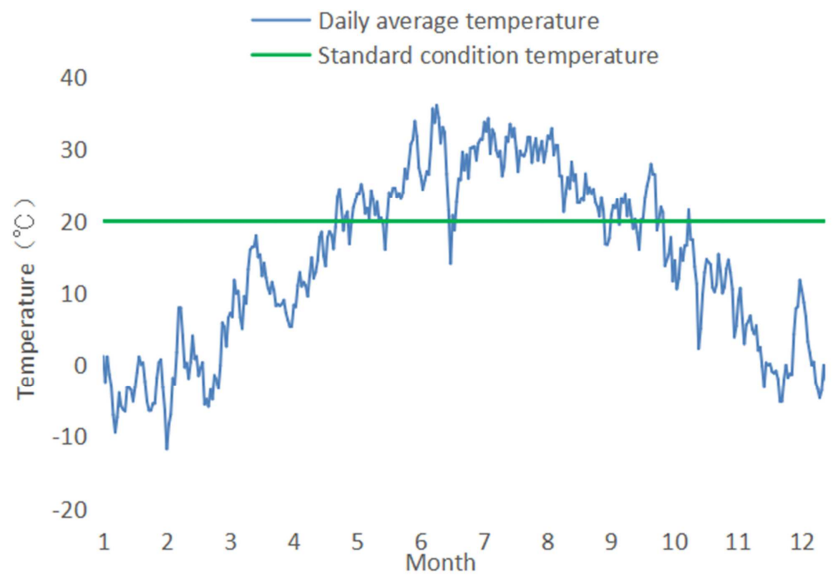

Figure 2. Annual temperature.

It can be seen from the trend line that the annual temperature in Jinan changes greatly, and the daily average minimum temperature can reach $-11^{\circ} \mathrm{C}$ and the maximum temperature can reach $34^{\circ} \mathrm{C}$. The average temperature from May to September is higher than the standard condition 
temperature, and the temperature from October to April is lower than the standard condition temperature. and the monthly average temperature is calculated by adding the hourly temperature of each month, which is used as the monthly average gas temperature of overhead courtyard pipeline, as shown in Table 1.

Table 1. Monthly average gas temperature of overhead courtyard pipeline.

\begin{tabular}{ll}
\hline Month & Gas temperature $\left({ }^{\circ} \mathbf{C}\right)$ \\
\hline January & -3.32 \\
February & -1.58 \\
March & 10.09 \\
April & 13.17 \\
May & 21.89 \\
June & 27.79 \\
July & 30.34 \\
August & 27.98 \\
September & 21.36 \\
October & 16.88 \\
November & 8.29 \\
December & 0.85 \\
\hline
\end{tabular}

It can be seen that the gas temperature of overhead pipeline varies greatly with seasons. The monthly average minimum temperature is $-3.32^{\circ} \mathrm{C}$, the monthly average maximum temperature can reach $30.34^{\circ} \mathrm{C}$, and the annual average temperature of gas in overhead pipeline is $14.48^{\circ} \mathrm{C}$.

\subsection{Gas Temperature of Buried Courtyard Pipeline}

Considering the pressure bearing capacity of the pipeline in the urban gas design, it is specified that the minimum covering thickness of the buried underground gas pipeline is: the motorway shall not be less than $0.9 \mathrm{~m}$, the bicycle lane shall not be less than $0.6 \mathrm{~m}$, and the place where it is impossible for the motor vehicle to reach shall not be less than $0.3 \mathrm{~m}$ [9]. The monthly average temperature variation of soil at different depths is shown in Figure 3:

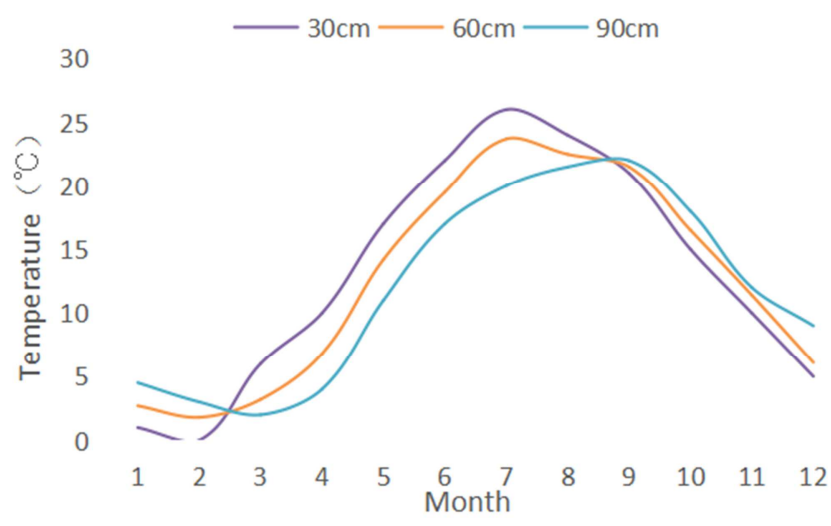

Figure 3. Monthly average temperature variation of soil.

The depth of 0.6 and $0.9 \mathrm{~m}$ is below the frozen soil layer, the temperature is always higher than $0^{\circ} \mathrm{C}$, and the fluctuation range of soil temperature is less than that of air temperature. In a year, the difference between the maximum monthly average temperature and the minimum monthly average temperature of soil is called the annual difference of soil temperature. The annual range of soil temperature decreases with the increase of depth. For soil, solar radiant energy is the main source of heat. The soil temperature changes with seasons. Compared with the temperature in the same period, the soil temperature is higher than the temperature in autumn and winter and lower than the temperature in spring and summer.

Take $0.6 \mathrm{~m}$ as the buried depth of courtyard pipeline in $\mathrm{J}$ City, and the monthly average temperature of soil at $0.6 \mathrm{~m}$ as the gas temperature of buried courtyard pipelines [10], as shown in Table 2.

Table 2. Monthly average gas temperature of buried courtyard pipeline.

\begin{tabular}{ll}
\hline Month & Gas temperature $\left({ }^{\circ} \mathbf{C}\right)$ \\
\hline January & 2.7 \\
February & 1.8 \\
March & 3.2 \\
April & 6.8 \\
May & 14.2 \\
June & 19.5 \\
July & 23.7 \\
August & 22.5 \\
September & 21.5 \\
October & 16.5 \\
November & 11.4 \\
December & 6.1 \\
\hline
\end{tabular}

The soil at $0.6 \mathrm{~m}$ is located below the frozen soil layer, the temperature is always higher than $0^{\circ} \mathrm{C}$, and the temperature fluctuation range is small. Compared with the gas temperature of outdoor courtyard pipeline overhead in the same period, the gas temperature of buried laying is higher than that of overhead laying in autumn and winter, and lower than that of overhead laying in spring and summer. The annual average temperature of buried gas is $12.49^{\circ} \mathrm{C}$.

\section{Error Analysis of Gas Meter}

\subsection{Calculation of Relative Error}

The volume of the gas measuring chamber of the gas meter is a fixed value, and the counter increases the standard condition value of the rotating volume every measurement. However, due to the influence of temperature, there is a gap between the actual working condition volume and the standard condition volume of gas, resulting in measurement error. Residential users use low-pressure pipeline to transmit gas. The gas supply pressure of residential users in $\mathrm{J}$ city is $2.4 \mathrm{KPa}$, less than $0.1 \mathrm{MPa}$. It is not necessary to consider the compensation and correction of the gas compression factor in the process of gas metering [11]. At this time, the gas can be regarded as an ideal gas. According to the ideal gas state equation, formula (1) is obtained:

$$
\frac{\mathrm{P}_{0} \mathrm{~V}_{0}}{\mathrm{~T}_{0}}=\frac{\mathrm{P}_{1} \mathrm{~V}_{1}}{\mathrm{~T}_{1}}
$$

Where $\mathrm{P}_{0}$ is absolute gas pressure in standard state, taken as $101325 \mathrm{~Pa}, \mathrm{~V}_{0}$ is gas volume in standard state, $\mathrm{T}_{0}$ is standard state temperature, $\mathrm{P}_{1}$ is absolute gas pressure in actual state, $V_{1}$ is gas volume in working state, $T_{1}$ is gas 
temperature in working state.

The relative error of the gas meter can be calculated by formula (2):

$$
\delta=\frac{\mathrm{V}_{1}-\mathrm{V}_{0}}{\mathrm{~V}_{0}}
$$

Where $\delta$ - Relative error of gas meter measurement

Substitute formula (1) into formula (2) to obtain the relationship between relative metering error and gas temperature and pressure. See formula (3):

$$
\delta=\frac{\mathrm{T}_{1}}{\mathrm{~T}_{0}} \frac{\mathrm{P}_{0}}{\mathrm{P}_{1}}-1
$$

That is, relative to the temperature and pressure in the standard state, the gas meter will produce a relative measurement error of $0.3 \%$ for every $1{ }^{\circ} \mathrm{C}$ change in the gas temperature in the actual state, and $1 \%$ for every $1 \mathrm{kPa}$ deviation of the pressure in the actual state from the standard condition pressure. The gas supply pressure is relatively stable in a year, but the gas temperature varies greatly with seasons. Replace the gas temperature in Table 1 for overhead laying and table 2 for buried laying into formula (3) to calculate the relative error of gas meter measurement per month under different laying modes of outdoor courtyard pipelines, as shown in table 3 .

Table 3. Relative error of gas meter.

\begin{tabular}{lll}
\hline Month & overhead pipelines\% & Buried Pipelines\% \\
\hline January & -8.79 & -6.75 \\
February & -8.20 & -7.06 \\
March & -4.25 & -6.58 \\
April & -3.21 & -5.37 \\
May & -0.27 & -2.87 \\
June & 1.73 & -1.07 \\
July & 2.59 & 0.35 \\
August & 1.79 & -0.06 \\
September & -0.44 & -0.40 \\
October & -1.96 & -2.09 \\
November & -4.86 & -3.81 \\
December & -7.38 & -5.60 \\
\hline
\end{tabular}

Summing the measurement relative error of the gas meter of the overhead courtyard pipeline and taking the average, the annual average measurement relative error is $\delta_{\mathrm{j}}=-2.77 \%$. Similarly, after summing the measurement relative error of the gas meter of the buried courtyard pipeline, the annual average measurement relative error is $\delta_{\mathrm{m}}=-3.44 \%$, the annual average measurement error of buried pipeline is greater than that of overhead pipeline, and the error increases with the increase of pipeline buried depth. This is because the soil has huge thermal melting and conduction resistance, and it is difficult to transfer heat to the underlying soil. Therefore, the deeper the depth, the lower the average annual soil temperature and the lower the gas temperature in the pipeline, the greater deviation from the standard temperature, the gas temperature greater by the meter error caused. The gas in the overhead pipeline is greatly affected by the temperature, absorbs the heat of solar radiation, and the temperature is high. The positive deviation in summer is much greater than that of the buried pipeline, offsetting most of the negative deviation in winter. Therefore, the measurement error caused by the gas temperature in the overhead pipeline is less than that of the gas temperature in the buried pipeline.

\subsection{Calculation of Annual Measurement Error}

Referring to the statistical yearbook of J City, the total annual gas consumption measured by gas meters of residential users in 2019 was $4.69 \times 10^{8} \mathrm{~m}^{3}$, During the reconstruction of some old residential areas in J City, due to conditions and other reasons, outdoor courtyard pipelines are overhead, and most rural coal to gas pipelines are overhead laid. The gas consumption of this part of users accounts for $40 \%$ of the total annual gas consumption, and the outdoor courtyard pipelines of other users are buried. Calculate the annual measurement error of gas meter according to formula (4).

$$
\Delta V=V_{b}\left(a \delta_{\mathrm{j}}+b \delta_{\mathrm{m}}\right)(i=1,2, \ldots, 12)
$$

Where $\Delta \mathrm{V}$ is annual measurement error of gas meter, $\mathrm{V}_{\mathrm{B}}$ is total annual gas consumption measured by gas meter of residential users, a is proportion of gas consumption of residential users of overhead pipeline, taking $a=0.4, b$ is proportion of gas consumption of residential users of buried pipelines, taking $b=0.6$.

It is calculated that the measurement error of the gas meter (i.e. the annual measurement error of the gas meter) caused by the deviation of the gas temperature from the standard state in $\mathrm{J}$ city is $-0.149 \times 10^{8} \mathrm{~m}^{3}$. When calculating the actual total gas loss, it is not only related to temperature, but also affects the gas consumption law of users. If it is considered that residential users use gas wall mounted furnace for heating in winter, the gas consumption is much higher than that in summer, which is the peak value of gas consumption in a year, and the measurement error of gas meter during this period is negative, the measurement error is greater than the calculated value of the above formula.

\subsection{Measurement Error Correction}

\subsubsection{Correction Factor}

At present, membrane gas meters are mostly used in domestic and catering gas measurement. The gas volume measurement is carried out by driving the left and right movement of the airbag endothelial membrane through the pressure difference between the inlet and outlet of the gas meter. The structure of gas meter is shown in Figure 1.

$$
V_{\mathrm{x}}=\gamma V_{\mathrm{b}}
$$

Where $\mathrm{V}_{\mathrm{x}}$ is corrected total annual gas consumption of residential users, $\mathrm{M}^{3}, \gamma$ is temperature correction factor.

The corrected total annual gas consumption is the difference between the total annual gas consumption displayed by the gas meter counter and the annual measurement error, as shown in formula (6):

$$
V_{\mathrm{x}}=V_{\mathrm{b}}-\Delta V
$$


After substituting equation (6) into equation (5), the calculation formula (7) of temperature correction coefficient is obtained:

$$
\gamma=1-\frac{\Delta V}{V_{\mathrm{b}}}
$$

When the gas temperature is lower than $20^{\circ} \mathrm{C}$, the gas meter counter shows that the gas consumption is less than the gas consumption converted into standard state, and the measurement error presents a negative difference, which will cause losses to the gas company, and the temperature correction coefficient is greater than 1 ; If the gas temperature is greater than $20^{\circ} \mathrm{C}$, the gas consumption displayed by the counter of the gas meter is more than that converted into the standard state, and the measurement error is positive, causing losses to the gas user. At this time, the correction coefficient is less than 1 .

The annual measurement error and the total annual gas consumption displayed by the gas meter counter are substituted into formula (7), and the temperature correction coefficient of $\mathrm{J}$ city is calculated as 1.032 . After correcting the total annual gas consumption displayed by the gas meter counter every year through formula (5), the loss caused by the metering error of the gas meter can be effectively reduced.

\subsubsection{Correction Technology}

With the development of technology and the attention of gas companies to measurement error, the measurement correction technology of gas meters continues to progress. Now the gas meters with volume correction function used in the market are mainly divided into mechanical and electronic types [12].

Because it is difficult to miniaturize the pressure sensor with mechanical pressure volume correction, it cannot be built into the gas meter. At present, there is no product with mechanical pressure volume correction technology in China. The mechanical temperature volume correction technology uses thermal bimetallic spring as the temperature sensitive element, and the thermal bimetallic is composed of two metal materials with different thermal ductility, Thermal sensitive metal materials will bend with temperature changes, store and release bending deformation energy, adjust the stroke of diaphragm of membrane gas meter through mechanical structure, and compensate the measurement error caused by temperature by changing the rotation volume of measurement system [13]. The thermal metal material is installed in the transmission system of the gas meter, and the metering correction can also be carried out by changing the transmission ratio. Mechanical temperature volume correction technology has the advantages of stable performance, high cost performance and less faults.

Electronic correction technology sets electronic equipment such as temperature sensor in the meter, measures the actual gas temperature passing through the gas meter, and designs the software algorithm according to the gas state equation, so that the intelligent operation unit of the gas meter can obtain the natural gas volume in the standard state through operation, so as to realize the temperature volume correction of natural gas. The electronic temperature compensation membrane gas meter has high correction accuracy, good real-time correction effect and high theoretical data support [14].

On September 1, 2020, the group standard T / CGAs 011-2020 electronic temperature and pressure correction membrane gas meter of China Urban Gas Association was implemented. The electronic temperature and pressure correction membrane gas meter is mainly used for the measurement of end users of gas companies, changing the original working condition into standard condition, which can reduce the gas loss at the end of the pipe network of gas companies, so as to improve the management efficiency of gas companies. [15]

\section{Conclusion}

Through the analysis of the gas temperature when the outdoor courtyard pipes are overhead and buried in J City, discussing the relative errors and temperature correction coefficients of the gas meters in J City by theoretical calculation methods. The main conclusions are as follows:

1. The annual average gas temperature of buried courtyard pipelines is lower than overhead, and the proportion of buried pipelines is relatively large. The measurement error caused by the deviation between actual working conditions and standard working conditions during buried laying is greater than that during overhead laying.

2. The annual average temperature of the gas in the overhead and buried pipelines both lower than $20^{\circ} \mathrm{C}$, the measurement error of the gas meter is negative The monthly gas consumption of residential users is uneven. When the gas temperature is low and the relative error is large in winter, the gas consumption increases due to the use of gas heating, resulting in more negative metering errors.

3. The negative measurement error of the gas meter causes losses to the gas company. For domestic gas meters without temperature compensation, the product of the total annual gas consumption displayed by the gas meter counter and the temperature correction coefficient should be used as the total annual gas consumption converted into the standard state. For city $\mathrm{J}$, the correction coefficient is 1.024 , which can reduce the production and sales difference of the gas company after correction.

According to the quality level and service status of membrane gas meters currently used in J City, it is recommended to conduct safety assessment on meters through necessary sampling inspection, combined with the overall repair rate and air leakage rate of residential users, properly replace and repair gas meters, reasonably save social resources and contribute to the realization of China's "carbon peak" goal. [16] 


\section{References}

[1] The General Administration of Quality Supervision, Inspection and Quarantine of the People's Republic of China and the National Standardization Administration of the People's Republic of China. Natural gas standard reference conditions: GB/T 19205-2008 [S]. 2008.

[2] Wang Xingfang, Li Hengjing, and Yan Yijian. The influence of pressure and temperature in front of a natural gas stove on the supply-sale gap [J]. Gas and Heat, vol. 2007, 27 (10), pp. 46-49.

[3] Wu Yuanyuan, Wenming, Wei Yong. The influence of temperature and pressure compensation on the measurement error of natural gas residents [J]. Gas and Heat, vol. 2016, 36 (7), pp. B17-B21

[4] Yan Congjie. Comparing two methods for evaluating the uncertainty of the indicated value error of membrane gas and discussing the role of sensitivity coefficient in uncertainty evaluation [J]. Measurement and testing technology, vol. 2021, 48 (4), pp. 109-112.

[5] Ma Shiren, Feng Zhigang. Measurement performance test of temperature compensated membrane gas meter [J]. China standardization, vol. 2021 (10), pp. 166-168+ 172.

[6] Jiang Hangcheng, Guo Gang, Lin Mingxing. Automatic calibration technology of intelligent membrane gas meter [J]. Gas and heat, vol. 2020, 40 (12), pp. 26-30 + 46 .

[7] Zhao Yumei. Study on rapid error verification method of membrane gas meter $[\mathrm{J}]$. Instrument standardization and measurement, vol. 2018 (2), pp. 35-37.
[8] Wang Lin. Research on Accurate Measurement Method of Gas Consumption for Catering Commercial Users (Master's Thesis) [D]. Harbin: Harbin Institute of Technology, 2017, pp. 11-15.

[9] Editor-in-Chief of the Ministry of Construction of the People's Republic of China. Urban Gas Design Code [S]. Beijing: China Construction Industry Press, 2006, pp. 394.

[10] Hai Chunxing. Chen Jianfei. Soil Geography [M]. Second edition. Beijing: Science Press, 2016, pp. 49.

[11] Li Chaohui, Dai Jingmin, Li Chengwei, etc. Discussion on the correction of compression factor in gas flow measurement [J]. Natural Gas Industry, vol. 2004, 24 (10), pp. 124-127.

[12] Liang Yongzeng. Technical analysis of electronic temperature and pressure correction membrane gas meter [J]. Gas and Heat, vol. 2019, 39 (11), pp. 33-36+46.

[13] Wang Haifang, Yu Hang, Li Shuwang, Yao Haiyu, Zhang Yaling, Wang Changlin, Wang Jian, Gao Zhu. Temperature and pressure compensation of diaphragm gas meter [J]. Gas and Heat, vol. 2012, 32 (01), pp. 63-65+81.

[14] Wang Xiuqiao, Li Hongjun, Zhao Xiaojun, Fang Jiong, Hu Liangchuan. Technical Research on Intelligent Ultrasonic Gas Meter [J]. Gas and Heat, vol. 2017, 37 (08), pp. 26-28.

[15] Yang Youtao, Peng Lei, Wang Zhen, Chen Shiyan. Theoretical basis and application of electronic temperature and pressure correction membrane gas meter [J]. Urban gas, vol. 2021 (02), pp. 1-4.

[16] Sun Junfang, Chen Tingting, Wang Xun, Chai Jiafeng, Tian Silu. Safety and metering performance analysis of civil membrane gas meters in use [J]. Modern occupational safety, vol. 2021 (08), pp. 89-92. 